\title{
Numerical Solution of Multidimensional Stochastic Itô-Volterra Integral Equation Based on the Least Squares Method and Block Pulse Function
}

\author{
Ting Ke ${ }^{(D)}$, Guo Jiang $(\mathbb{D}$, and Mengting Deng $(\mathbb{C}$ \\ School of Mathematics and Statistics, Hubei Normal University, Huangshi 435002, Hubei, China \\ Correspondence should be addressed to Guo Jiang; gjiang@hbnu.edu.cn
}

Received 8 November 2020; Revised 6 February 2021; Accepted 9 February 2021; Published 24 February 2021

Academic Editor: Łukasz Jankowski

Copyright ( 2021 Ting Ke et al. This is an open access article distributed under the Creative Commons Attribution License, which permits unrestricted use, distribution, and reproduction in any medium, provided the original work is properly cited.

\begin{abstract}
In this paper, a method based on the least squares method and block pulse function is proposed to solve the multidimensional stochastic Itô-Volterra integral equation. The Itô-Volterra integral equation is transformed into a linear algebraic equation. Furthermore, the error analysis is given by the isometry property and Doob's inequality. Numerical examples verify the effectiveness and precision of this method.
\end{abstract}

\section{Introduction}

Stochastic Volterra integral equations have been generally used in many fields such as mechanics, medicine, materials, finance, and physical science, especially in the process of engineering practice, because many material coefficients are uncertain, it is necessary to introduce stochastic integral equations to establish models. Meanwhile, the stochastic integral equation is used to solve a variety of engineering problems, for example, material science modeling [1], automated systems science [2], dynamics [3], and biology [4]. However, most of the stochastic Volterra integral equations cannot be solved explicitly. Therefore, it is essential to provide numerical solutions to these equations. Many scholars have developed various methods to study the stochastic Volterra integral equation. Different Volterra integral equations are handled by Bernstein polynomials, block pulse functions, least squares method, Haar wavelets, Walsh functions, Chebyshev and Legendre polynomials, and other functions. We only mentioned the referenced such as [5-21] and other relevant literatures. On the other hand, some authors obtained the numerical solution of stochastic Volterra integral equation by Euler-Maruyama approximation or iterative algorithm, for example [22-27].

Recently, there have been many articles on the numerical solution of the stochastic Itô-Volterra integral equation. For example, in [13], Maleknejad et al. considered linear stochastic Itô-Volterra integral equations (SIVIEs) by block pulse functions (BPFs). In [15], Wu et al. used Haar wavelets (HWs) to solve nonlinear SIVIEs. Mirzaee et al. studied nonlinear SIVIEs of fractional order based on the hybrid of block pulse and parabolic functions [16]. In [28], Jiang et al. used BPFs to obtain the numerical solution of two-dimensional nonlinear SIVIEs. Ahmadinia et al. presented a new method that applies the least squares method to study SIVIEs [29]. Moreover, Maleknejad et al. simplified m-dimensional SIVIEs into linear lower trigonometric equations by using BPFs and stochastic integral operation matrix, and then the equations were solved [30].

Motivated by the abovementioned literatures, we consider the following multidimensional linear SIVIEs which are studied relatively little and propose a method that combines BPFs and least squares method. 


$$
\begin{aligned}
X(t)= & f(t)+\int_{0}^{t} \widetilde{S}(u, t) X(u) \mathrm{d} u \\
& +\sum_{k=1}^{q} \int_{0}^{t} \widehat{S}_{k}(u, t) X(u) \mathrm{d} B_{k}(u), \quad t \in[0, T),
\end{aligned}
$$

where $X(t), \widetilde{S}(u, t)$, and $\widehat{S}_{k}(u, t), k=1,2, \ldots, q$, for $0<u<t$, are stochastic processes on the same probability space of $(\Omega, \mathscr{F}, P), f(t)$ is an initial function, and $X(t)$ is unknown. $B_{k}(u)$ are Brownian motions and $\int_{0}^{t} \widehat{S}_{k}(u, t) X(u) \mathrm{d} B_{k}(u)$ are Itô integrals.

In contrast to the articles $[13,29]$, this paper's difference is to study linear SIVIEs driven by multiple independent Brownian motions. In addition, the numerical solution based on the least squares method and BPFs is more accurate than other methods in the reference [30]. Finally, this method can be applied to the models of engineering and material science, which makes the practical data more meaningful.

In Section 2, BPFs are introduced. In Section 3, stochastic integral operation matrix is given. In Section 4 , the least squares method is recommended. In Section 5, the error analysis is obtained. In Section 6, the accuracy of the method is verified by two numerical examples. In Section 7 , the conclusion is given.

\section{Preliminaries}

Definition 1 (see [31]) (BPFs).

BPFs have been considered and used to solve various equations by numerous scholars, such as $[8,12,13,28,30]$. BPFs are denoted as

$$
\psi_{i}(t)= \begin{cases}1, & (i-1) h \leq t<i h, \\ 0, & \text { otherwise }\end{cases}
$$

where $t \in[0, T), i=1, \ldots, m$, and $h=T / m$.

The basic properties of BPFs are described as follows:

(1) Disjointness:

$$
\psi_{i}(t) \psi_{j}(t)=\delta_{i j} \psi_{i}(t), \quad i, j=1,2, \ldots, m,
$$

where $\delta_{i j}$ is the Kronecker delta.

(2) Orthogonality:

$$
\int_{0}^{T} \psi_{i}(t) \psi_{j}(t) \mathrm{d} t=h \delta_{i j}
$$

(3) Completeness: Parseval's identity is true for every $f \in L^{2}([0, T))$ and $f(t)=\sum_{i=1}^{\infty} f_{i} \psi_{i}(t)$,

$$
\int_{0}^{T} f^{2}(t) \mathrm{d} t=\sum_{i=1}^{\infty} f_{i}^{2}\left\|\psi_{i}(t)\right\|
$$

where $f_{i}=(1 / h) \int_{0}^{T} f(t) \psi_{i}(t) \mathrm{d} t$.

BPFs are expressed as a vector

$$
\Psi(t)=\left(\psi_{1}(t), \psi_{2}(t), \ldots, \psi_{m}(t)\right)^{T}
$$

It can be concluded from the above description that

$$
\begin{aligned}
\Psi_{m}(t) \Psi_{m}^{T}(t) & =\left(\begin{array}{cccc}
\psi_{1}(t) & 0 & \cdots & 0 \\
0 & \psi_{2}(t) & \cdots & 0 \\
\vdots & \vdots & \ddots & 0 \\
0 & 0 & \cdots & \psi_{m}(t)
\end{array}\right)_{m \times m}, \\
\Psi_{m}^{T}(t) \Psi_{m}(t) & =1, \\
\Psi_{m}(t) \Psi_{m}^{T}(t) F_{m} & =D_{F_{m}} \Psi_{m}(t) .
\end{aligned}
$$

where $F_{m}=\left(f_{1}, f_{2}, \ldots, f_{m}\right)^{T}$ and $\mathbf{D}_{F_{m}}=\operatorname{diag}\left(F_{m}\right)$.

Any function $X(t) \in L^{2}([0, T))$ can be approximately expressed as

$$
\begin{aligned}
X(t) & \simeq X_{m}(t)=\sum_{i=1}^{m} c_{i} \psi_{i}(t)=\Psi_{m}^{T}(t) C_{m}, \\
C_{m} & =\left(c_{1}, c_{2}, \ldots, c_{m}\right)^{T},
\end{aligned}
$$

where $X_{m}(t)$ is a linear combination of BPFs.

Let $S(u, t) \in L^{2}\left(\left[0, T_{1}\right) \times\left[0, T_{2}\right)\right)$; it can be extended as

$$
S(u, t)=\Psi_{m_{1}}^{T}(u) \mathbf{S} \Psi_{m_{2}}(t)=\Psi_{m_{2}}^{T}(t) \mathbf{S}^{T} \Psi_{m_{1}}(u),
$$

where $\mathbf{S}=\left(s_{i j}\right)_{m_{1} \times m_{2}}$,

$$
s_{i j}=\frac{1}{h_{1} h_{2}} \int_{0}^{T_{1}} \int_{0}^{T_{2}} S(u, t) \psi_{i}(u) \phi_{j}(t) \mathrm{d} u \mathrm{~d} t,
$$

and $h_{1}=\left(T_{1} / m_{1}\right), h_{2}=\left(T_{2} / m_{2}\right)$. In order to facilitate, we put $m_{1}=m_{2}=m$ in the following sections.

\section{Stochastic Integration Operational Matrix}

The section gives the relevant lemmas.

Lemma 1 (see $[8,13]$ ). Supposing $\Psi_{m}(t)$ is given in (6), we obtain

$$
\int_{0}^{t} \Psi_{m}(u) \mathrm{d} u \simeq \mathbf{Q} \Psi_{m}(t)
$$

where

$$
\mathbf{Q}=\frac{h}{2}\left(\begin{array}{ccccc}
1 & 2 & 2 & \cdots & 2 \\
0 & 1 & 2 & \cdots & 2 \\
0 & 0 & 1 & \cdots & 2 \\
\vdots & \vdots & \vdots & \ddots & \vdots \\
0 & 0 & 0 & \cdots & 1
\end{array}\right)_{m \times m} .
$$

Therefore, each function $X(t) \in L^{2}([0, T))$ can be approximately expressed as

$$
\int_{0}^{t} X(u) \mathrm{d} u \simeq C_{m}^{T} \mathbf{Q} \Psi_{m}(t)
$$


Lemma 2 (see $[8,13]$ ). Supposing $\Psi_{m}(t)$ is given in (6), we obtain

$$
\int_{0}^{t} \Psi_{m}(u) \mathrm{d} B(u) \simeq \mathbf{Q}_{B} \Psi_{m}(t),
$$

where $\mathbf{Q}_{B}$ can be expressed as

$$
\mathbf{Q}_{B}=\left(\begin{array}{ccccc}
\beta_{1} & \alpha_{1} & \alpha_{1} & \cdots & \alpha_{1} \\
0 & \beta_{2} & \alpha_{2} & \cdots & \alpha_{2} \\
0 & 0 & \beta_{3} & \cdots & \alpha_{3} \\
\vdots & \vdots & \vdots & \ddots & \vdots \\
0 & 0 & 0 & \cdots & \beta_{m}
\end{array}\right)_{m \times m},
$$

where $\quad \alpha_{i}=B(i h)-B((i-1) h), i=1,2, \ldots, m-1$; $\beta_{j}=B(i h / 2)-B((i-1) h / 2), j=1,2, \ldots, m$.

Therefore, each function $X(t) \in L^{2}([0, T))$ can be approximately expressed as

$$
\int_{0}^{t} X(u) \mathrm{d} B(u) \simeq C_{m}^{T} \mathbf{Q}_{B} \Psi_{m}(t) .
$$

\section{Method Description}

In this section, the multidimensional linear SIVIEs are transformed into linear algebraic equations by the least square method and BPFs.

The linear operator $L$ is taken into account,

$$
\begin{aligned}
L(X(t)):= & X(t)-\int_{0}^{t} \widetilde{S}(u, t) X(u) \mathrm{d} u \\
& -\sum_{k=1}^{q} \int_{0}^{t} \widehat{S}_{k}(u, t) X(u) \mathrm{d} B_{k}(u) .
\end{aligned}
$$

If $X(t)$ is the exact solution to (1), then the residual norm disappears:

$$
\|L(X)-f\|_{L^{2}}=0 .
$$

For $\varepsilon>0, X_{\varepsilon}(t)$ is the approximate solution, so that the residual norm of $X_{\varepsilon}(t)$ is less than $\varepsilon$,

$$
\left\|X_{\varepsilon}(t)-f\right\|_{L^{2}}<\varepsilon
$$

An approximate solution can be obtained by (19), and $X_{m}$ is a linear combination by BPFs,

$$
X_{m}=\sum_{i=1}^{m} c_{i} \psi_{i}(t)
$$

where $c_{i}$ is the unknown coefficient.

The minimization problem is studied below,

$$
\min _{c_{1}, \ldots, c_{m}}\left\|L\left(X_{m}\right)-f\right\|_{L^{2}}
$$

Minimizing (21) to get a set of values of $\widehat{c}_{1}, \ldots, \widehat{c}_{m}$, then $\sum_{i=1}^{m} \widehat{c}_{i} \psi_{i}(t)$ is an approximate solution to (1), and we'll prove it.

$$
\begin{aligned}
& \mathbb{E}\left[\min _{c_{1}, \ldots, c_{m}}\left\|L\left(X_{m}\right)-f\right\|_{L^{2}}\right] \\
& =\mathbb{E}\left[\left\|L\left(\sum_{i=1}^{m} \widehat{c}_{i} \psi_{i}\right)-f\right\|_{L^{2}}\right] \longrightarrow 0,
\end{aligned}
$$

where $m \longrightarrow \infty$ (or $h \longrightarrow 0)$.

To obtain the minimum value of the approximate solution in (21), we have to take the partial derivative in $c_{i}$, and let

$$
\frac{\partial}{\partial c_{i}} \int_{0}^{T}\left(\sum_{j=1}^{m} c_{j} L\left(\psi_{j}(t)\right)-f(t)\right)^{2} \mathrm{~d} t=0
$$

where $i=1,2, \ldots, m$.

Then,

$$
\begin{gathered}
\int_{0}^{T} \sum_{j=1}^{m} c_{j} L\left(\psi_{j}(t)\right) L\left(\psi_{i}(t)\right) \mathrm{d} t \\
=\int_{0}^{T} f(t) L\left(\psi_{i}(t)\right) \mathrm{d} t,
\end{gathered}
$$

that is,

$$
\sum_{j=1}^{m} c_{j}\left\langle L\left(\psi_{i}(t)\right), L\left(\psi_{j}(t)\right)\right\rangle=\left\langle L\left(\psi_{i}(t)\right), f(t)\right\rangle,
$$

where $\left\langle L\left(\psi_{i}(t)\right), L\left(\psi_{j}(t)\right)\right\rangle:=\int_{0}^{T} L\left(\psi_{j}(t)\right) L\left(\psi_{i}(t)\right) \mathrm{d} t$.

The following is expressed as a matrix:

$$
c=\mathbf{G}^{-1} a
$$

where $\quad c=\left(c_{1}, c_{2}, \ldots, c_{m}\right)^{T} \in \mathbb{R}^{m}, a=\left(a_{1}, a_{2}, \ldots, a_{m}\right)^{T} \in$ $\mathbb{R}^{m}$, and $\mathbf{G}=\left(g_{i j}\right) \in \mathbb{R}^{m \times m}$ such that

$$
\begin{aligned}
a_{i} & =\left\langle L\left(\psi_{i}(t)\right), f(t)\right\rangle, \\
g_{i j} & =\left\langle L\left(\psi_{i}(t)\right), L\left(\psi_{j}(t)\right)\right\rangle, \quad i, j=1,2, \ldots, m .
\end{aligned}
$$

For solving equation (1), $X(t), f(t), \widetilde{S}(u, t)$, and $\widehat{S}(u, t)$ can be approximated by BPFs in the following forms:

$$
\begin{gathered}
X(t) \simeq X_{m}(t)=C_{m}^{T} \Psi_{m}(t)=\Psi_{m}^{T}(t) C_{m}, \\
f(t) \simeq f_{0}(t)=F_{m}^{T} \Psi_{m}(t)=\Psi_{m}^{T}(t) F_{m}, \\
\widetilde{S}(u, t)=\Psi_{m}^{T}(u) \mathbf{S}_{1} \Psi_{m}(t)=\Psi_{m}^{T}(t) \mathbf{S}_{1}^{T} \Psi_{m}(u), \\
\widehat{S}(u, t)=\Psi_{m}^{T}(u) \mathbf{S}_{2} \Psi_{m}(t)=\Psi_{m}^{T}(t) \mathbf{S}_{2}^{T} \Psi_{m}(u),
\end{gathered}
$$

where $C_{m}$ and $F_{m}$ are BPF coefficient vectors; $\mathbf{S}_{1}$ and $\mathbf{S}_{2}$ are BPF coefficient matrices by the equation of (10).

Next, for obtaining $a_{i}$ and $g_{i j}$, by (17) and (28)-(31), we have 


$$
\begin{aligned}
L\left(\psi_{i}(t)\right)= & \psi_{i}(t)-\int_{0}^{t} \widetilde{S}(u, t) \psi_{i}(u) \mathrm{d} u \\
& -\sum_{k=1}^{q} \int_{0}^{t} \widehat{S}_{k}(u, t) \psi_{i}(u) \mathrm{d} B_{k}(u) \\
= & I_{i} \Psi_{m}(t)-\int_{0}^{t} I_{i} \Psi_{m}(u) \Psi_{m}^{T}(u) \mathbf{S}_{1} \Psi_{m}(t) \mathrm{d} u \\
& -\sum_{k=1}^{q} \int_{0}^{t} I_{i} \Psi_{m}(u) \Psi_{m}^{T}(u) \mathbf{S}_{2} \Psi_{m}(t) \mathrm{d} B_{k}(u) \\
= & I_{i} \Psi_{m}(t)-I_{i} \int_{0}^{t} \Psi_{m}(u) \Psi_{m}^{T}(u) \mathrm{d} u \mathbf{S}_{1} \Psi_{m}(t) \\
& -I_{i} \sum_{k=1}^{q} \int_{0}^{t} \Psi_{m}(u) \Psi_{m}^{T}(u) \mathrm{d} B_{k}(u) \mathbf{S}_{2} \Psi_{m}(t),
\end{aligned}
$$

where $I_{i}$ is the ith row of an $m \times m$ identity matrix.

Let $S_{1}^{i}$ and $S_{2}^{i}$ be the ith row of the matrix $\mathbf{S}_{1}$ and $\mathbf{S}_{2}$, respectively, $R^{i}$ and $R_{B}^{i}$ be the ith row of the integral operation matrix $\mathbf{Q}$ and the stochastic integral matrix $\mathbf{Q}_{B}$, respectively, and $\mathbf{D}_{S_{1}^{i}}$ and $\mathbf{D}_{S_{2}^{i}}$ be a diagonal matrix with $S_{1}^{i}$ and $S_{2}^{i}$ as the diagonal elements, respectively. By (11) and (14), we get

$$
\begin{aligned}
& \left(\int_{0}^{t} \Psi_{m}(u) \Psi_{m}(u)^{T} \mathrm{~d} u\right) \mathbf{S}_{1} \Psi_{m}(t), \\
& =\left(\begin{array}{c}
R^{1} \Psi_{m}(t) S_{1}^{1} \Psi_{m}(t) \\
R^{2} \Psi_{m}(t) S_{1}^{2} \Psi_{m}(t) \\
\vdots \\
R^{m} \Psi_{m}(t) S_{1}^{m} \Psi_{m}(t)
\end{array}\right) \\
& =\left(\begin{array}{c}
R^{1} \mathbf{D}_{S_{1}^{1}} \\
R^{2} \mathbf{D}_{S_{1}^{2}} \\
\vdots \\
R^{m} \mathbf{D}_{S_{1}^{m}}
\end{array}\right) \Psi_{m}(t)=\widetilde{\mathbf{S}} \Psi_{m}(t),
\end{aligned}
$$

where

$$
\widetilde{\mathbf{S}}=\frac{h}{2}\left(\begin{array}{ccccc}
\widetilde{S}_{11} & 2 \widetilde{S}_{12} & 2 \widetilde{S}_{13} & \cdots & 2 \widetilde{S}_{1 m} \\
0 & \widetilde{S}_{22} & 2 \widetilde{S}_{23} & \cdots & 2 \widetilde{S}_{2 m} \\
0 & 0 & \widetilde{S}_{33} & \cdots & 2 \widetilde{S}_{3 m} \\
\vdots & \vdots & \vdots & \ddots & \vdots \\
0 & 0 & 0 & \cdots & \widetilde{S}_{m m}
\end{array}\right)_{m \times m} \text {, }
$$

$$
\begin{aligned}
& \left(\int_{0}^{t} \Psi_{m}(u) \Psi_{m}(u)^{T} \mathrm{~d} B(u)\right) \mathbf{S}_{2} \Psi_{m}(t) \\
& =\left(\begin{array}{c}
R_{B}^{1} \Psi_{m}(t) S_{2}^{1} \Psi_{m}(t) \\
R_{B}^{2} \Psi_{m}(t) S_{2}^{2} \Psi_{m}(t) \\
\vdots \\
R_{B}^{m} \Psi_{m}(t) S_{2}^{m} \Psi_{m}(t)
\end{array}\right) \\
& =\left(\begin{array}{c}
R_{B}^{1} \mathbf{D}_{S_{2}^{1}} \\
R_{B}^{2} \mathbf{D}_{S_{2}^{2}} \\
\vdots \\
R_{B}^{m} \mathbf{D}_{S_{2}^{m}}
\end{array}\right) \Psi_{m}(t)=\widehat{\mathbf{S}} \Psi_{m}(t),
\end{aligned}
$$

where

$$
\widehat{\boldsymbol{S}}_{k}=\left(\begin{array}{ccccc}
\widehat{S}_{11}^{k} \beta_{1} & \widehat{S}_{12}^{k} \alpha_{1} & \widehat{S}_{13}^{k} \alpha_{1} & \ldots & \widehat{S}_{1 m}^{k} \alpha_{1} \\
0 & \hat{S}_{22}^{k} \beta_{2} & \hat{S}_{23}^{k} \alpha_{2} & \ldots & \widehat{S}_{2 m}^{k} \alpha_{2} \\
0 & 0 & \widehat{S}_{33}^{k} \beta_{3} & \ldots & \widehat{S}_{3 m}^{k} \alpha_{3} \\
\vdots & \vdots & \vdots & \ddots & \vdots \\
0 & 0 & 0 & \cdots & \widehat{S}_{m m}^{k} \beta_{m}
\end{array}\right)_{m \times m} .
$$


According to Lemmas 1 and 2,

Then,

$$
\begin{aligned}
L\left(\psi_{i}(t)\right) & =I_{i} \Psi_{m}(t)-I_{i} \widetilde{\mathbf{S}} \Psi_{m}(t)-I_{i} \sum_{k=1}^{q} \widehat{\mathbf{S}}_{k} \Psi_{m}(t) \\
& =\left(I_{i}-I_{i} \widetilde{\mathbf{S}}-I_{i} \sum_{k=1}^{q} \widehat{\mathbf{S}}_{k}\right) \Psi_{m}(t) .
\end{aligned}
$$

$$
\begin{aligned}
& a_{i}=\left\langle L\left(\psi_{i}(t)\right), f(t)\right\rangle=\int_{0}^{T} L\left(\psi_{i}(t)\right) f(t) \mathrm{d} t \\
& =\int_{0}^{T}\left(I_{i}-I_{i} \widetilde{\mathbf{S}}-I_{i} \sum_{k=1}^{q} \widehat{\mathbf{S}}_{k}\right) \Psi_{m}(t) F_{m}^{T} \Psi_{m}(t) \mathrm{d} t \\
& =\int_{0}^{T}\left(I_{i}-I_{i} \widetilde{\mathbf{S}}-I_{i} \sum_{k=1}^{q} \widehat{\mathbf{S}}_{k}\right) F_{m} \Psi_{m}^{T}(t) \Psi_{m}(t) \mathrm{d} t \\
& =\left(I_{i}-I_{i} \widetilde{\mathbf{S}}-I_{i} \sum_{k=1}^{q} \widehat{\mathbf{S}}_{k}\right) F_{m} \int_{0}^{T} \Psi_{m}^{T}(t) \Psi_{m}(t) \mathrm{d} t \\
& =\left(I_{i}-I_{i} \widetilde{\mathbf{S}}-I_{i} \sum_{k=1}^{q} \widehat{\mathbf{S}}_{k}\right) F_{m} T, \\
& g_{i j}=\left\langle L\left(\psi_{i}(t)\right), L\left(\psi_{j}(t)\right)\right\rangle \\
& =\int_{0}^{T} L\left(\psi_{i}(t)\right) L\left(\psi_{j}(t)\right) \mathrm{d} t \\
& =\int_{0}^{T}\left(I_{i}-I_{i} \widetilde{\mathbf{S}}-I_{i} \sum_{k=1}^{q} \widehat{\mathbf{S}}_{k}\right) \Psi_{m}(t)\left(I_{j}-I_{j} \widetilde{\mathbf{S}}-I_{j} \sum_{k=1}^{q} \widehat{\mathbf{S}}_{k}\right) \Psi_{m}(t) \mathrm{d} t \\
& =\int_{0}^{T}\left(I_{i}-I_{i} \widetilde{\mathbf{S}}-I_{i} \sum_{k=1}^{q} \widehat{\mathbf{S}}_{k}\right)\left(I_{j}-I_{j} \widetilde{\mathbf{S}}-I_{j} \sum_{k=1}^{q} \widehat{\mathbf{S}}_{k}\right)^{T} \Psi_{m}^{T}(t) \Psi_{m}(t) \mathrm{d} t \\
& =\left(I_{i}-I_{i} \widetilde{\mathbf{S}}-I_{i} \sum_{k=1}^{q} \widehat{\mathbf{S}}_{k}\right) \\
& \cdot\left(I_{j}-I_{j} \widetilde{\mathbf{S}}-I_{j} \sum_{k=1}^{q} \widehat{\mathbf{S}}_{k}\right)^{T} \int_{0}^{T} \Psi_{m}^{T}(t) \Psi_{m}(t) \mathrm{d} t \\
& =\left(I_{i}-I_{i} \widetilde{\mathbf{S}}-I_{i} \sum_{k=1}^{q} \widehat{\mathbf{S}}_{k}\right)\left(I_{j}-I_{j} \widetilde{\mathbf{S}}-I_{j} \sum_{k=1}^{q} \widehat{\mathbf{S}}_{k}\right)^{T} T .
\end{aligned}
$$

\section{Error Analysis}

We will give the error analysis in this section.

Lemma 3 (see [29]) (continuous module). $f$ with respect to $\varrho$ in $[0, T]$ is the definition of continuous modulus $\omega(f, \varrho)$

$$
\begin{aligned}
& \omega(f, \varrho) \\
& =\sup \{|f(x)-f(y)| x, y \in[0, T],|x-y| \leq \varrho\} .
\end{aligned}
$$

As shown in the reference [32], if and only if $\lim _{\rho \longrightarrow 0} \omega(f, \varrho)=0$, on $[0, T], f(t)$ is uniformly continuous.
Lemma 4 (see [29]). Suppose $f \in C[0, T], Q_{h}=\sum_{i=1}^{m} e_{i} \psi_{i}(t)$, where $e_{i}=f\left(\left(t_{i-1}+t_{i}\right) / 2\right), t_{i}=i h, h=T / m, i=0,1, \ldots, m$. Then,

$$
\left\|f-Q_{h}\right\|_{\infty} \leq \omega(f, h)
$$

Theorem 1. Suppose $X(t)$ be the exact solution of (1) and $\widehat{X}_{m}(t)=\sum_{i=1}^{m} e_{i} \psi_{i}(t)$, where $e_{i}=X\left(\left(t_{i-1}+t_{i}\right) / 2\right), \widetilde{S}(u, t)$, and $\widehat{S}_{k}(u, t)$ are deterministic functions, $\|\widetilde{S}\|_{\infty} \leq M,\left\|\widehat{S}_{k}\right\|_{\infty} \leq M$, where $M$ is a positive constant. Then, we get the following conclusions, when $h \longrightarrow 0$ : 
TABLE 1: When $m=2^{4}$, the table shows error means $E_{m}$, error standard deviations $E_{s}$, and confidence intervals for different time $t$.

\begin{tabular}{cccrr}
\hline$t$ & \multirow{2}{*}{$E_{m}$} & $E_{s}$ & \multicolumn{2}{c}{$95 \%$ confidence interval for error mean } \\
& & & Lower & Upper \\
\hline $1 / 16$ & $1.36075252 \times 10^{-4}$ & $6.80376264 \times 10^{-5}$ & $2.7215050 \times 10^{-6}$ & $2.6942900 \times 10^{-4}$ \\
$3 / 16$ & $1.13092940 \times 10^{-4}$ & $5.65464702 \times 10^{-5}$ & $2.2618588 \times 10^{-6}$ & $2.2392402 \times 10^{-4}$ \\
$5 / 16$ & $5.33574672 \times 10^{-5}$ & $2.66787336 \times 10^{-5}$ & $1.0671493 \times 10^{-6}$ & $1.0564778 \times 10^{-4}$ \\
$7 / 16$ & $2.67128325 \times 10^{-5}$ & $1.33564162 \times 10^{-5}$ & $5.3425665 \times 10^{-7}$ & $5.2891408 \times 10^{-5}$ \\
$9 / 16$ & $2.53074880 \times 10^{-6}$ & $1.26537440 \times 10^{-6}$ & $5.0614976 \times 10^{-8}$ & $5.0108826 \times 10^{-6}$ \\
\hline
\end{tabular}

TABLE 2: When $m=2^{5}, n=100$, this table shows error means $E_{m}$, error standard deviations $E_{s}$, and confidence intervals for different time $t$.

\begin{tabular}{lccrr}
\hline$t$ & $E_{m}$ & $E_{s}$ & \multicolumn{2}{c}{$95 \%$ confidence interval for error mean } \\
& & & Lower & Upper \\
\hline $1 / 32$ & $1.47378183 \times 10^{-4}$ & $7.36890915 \times 10^{-5}$ & $2.9475636 \times 10^{-6}$ & $2.9180880 \times 10^{-4}$ \\
$7 / 32$ & $9.11708782 \times 10^{-5}$ & $4.55854391 \times 10^{-5}$ & $1.8234175 \times 10^{-6}$ & $1.8051833 \times 10^{-4}$ \\
$13 / 32$ & $5.04388689 \times 10^{-5}$ & $2.52194344 \times 10^{-5}$ & $1.0087773 \times 10^{-6}$ & $9.9868960 \times 10^{-5}$ \\
$19 / 32$ & $9.47544810 \times 10^{-6}$ & $4.73772405 \times 10^{-6}$ & $1.8950896 \times 10^{-7}$ & $1.8761387 \times 10^{-5}$ \\
$25 / 32$ & $4.47443350 \times 10^{-5}$ & $2.23721675 \times 10^{-5}$ & $8.9488670 \times 10^{-7}$ & $8.8593783 \times 10^{-5}$ \\
\hline
\end{tabular}

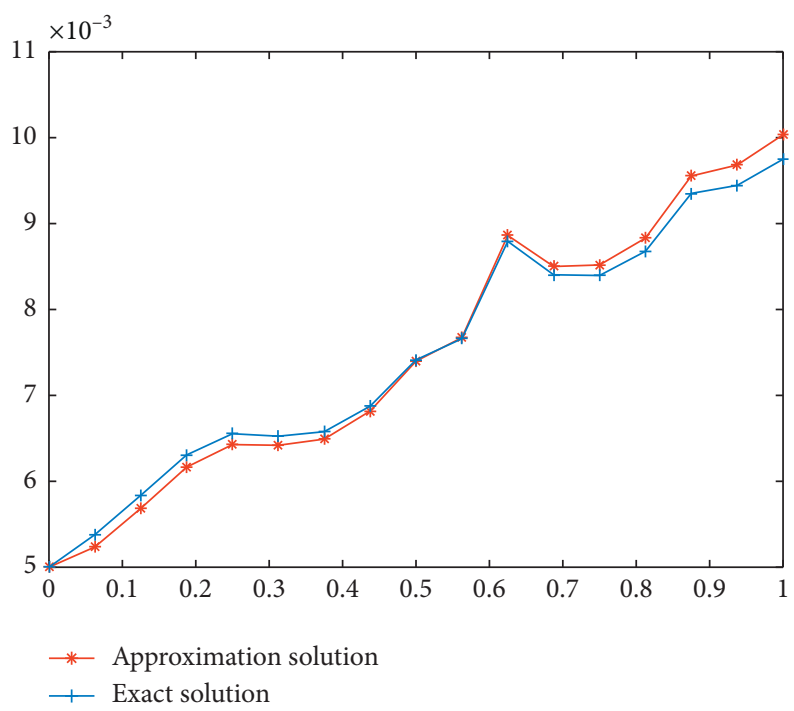

Figure 1: $m=2^{4}$, the approximate solution and exact solution for Example 1.

(i) $\left\|\widehat{X}_{m}(t)-X(t)\right\|_{\infty, E}=\mathbb{E}\left[\sup _{t \in[0, T)}\left|\widehat{X}_{m}(t)-X(t)\right|\right] \longrightarrow 0$,
(ii) $\mathbb{E}\left[\min _{c_{1}, \ldots, c_{m}}\left\|X_{m}(t)-f(t)-\int_{0}^{t} \widetilde{S}(u, t) X_{m}(u) \mathrm{d} u-\sum_{k=1}^{q} \int_{0}^{t} \widehat{S}_{k}(u, t) X_{m}(u) \mathrm{d} B_{k}(u)\right\|_{L^{2}}^{2}\right] \longrightarrow 0$.

Proof

(i) By Lemma 4, we have

$$
\begin{aligned}
& \left\|\widehat{X}_{m}(t)-X(t)\right\|_{\infty, E} \\
& =\mathbb{E}\left[\sup _{t \in[0, T)}\left|\widehat{X}_{m}(t)-X(t)\right|\right] \leq \omega(X, h) \longrightarrow 0 .
\end{aligned}
$$




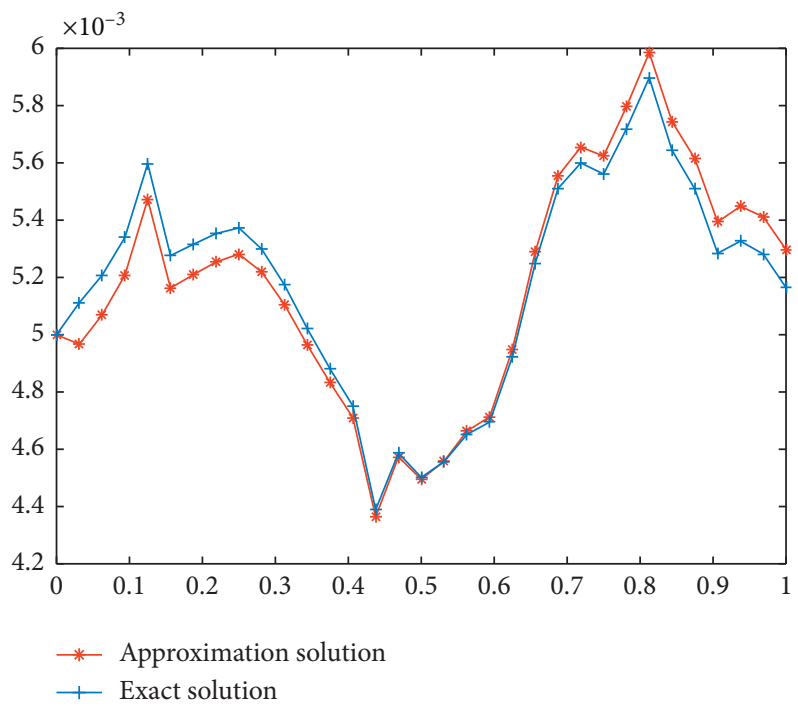

Figure 2: $m=2^{5}$, the approximate solution and exact solution for Example 1 .

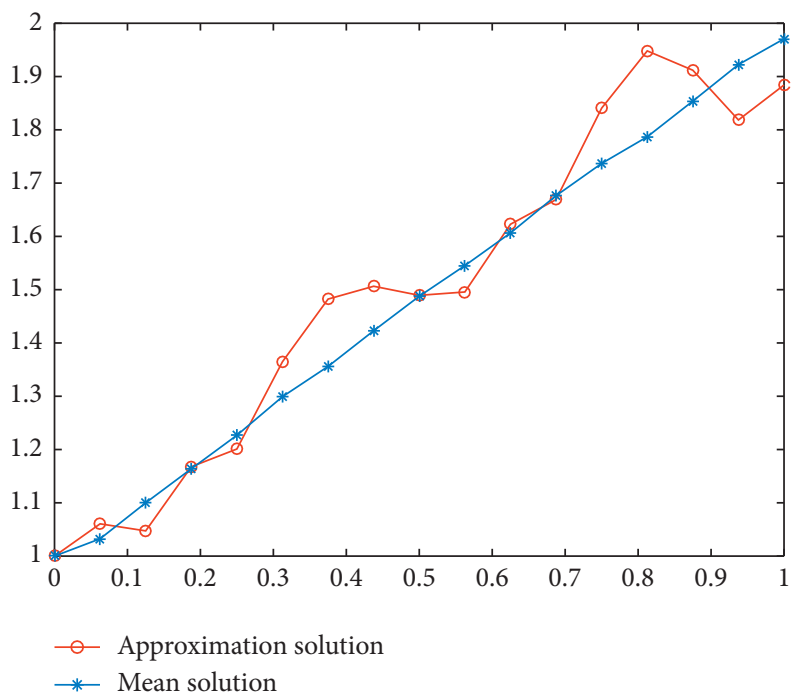

FIgURE 3: $m=2^{4}$, the approximate solution and mean solution for Example 2 .

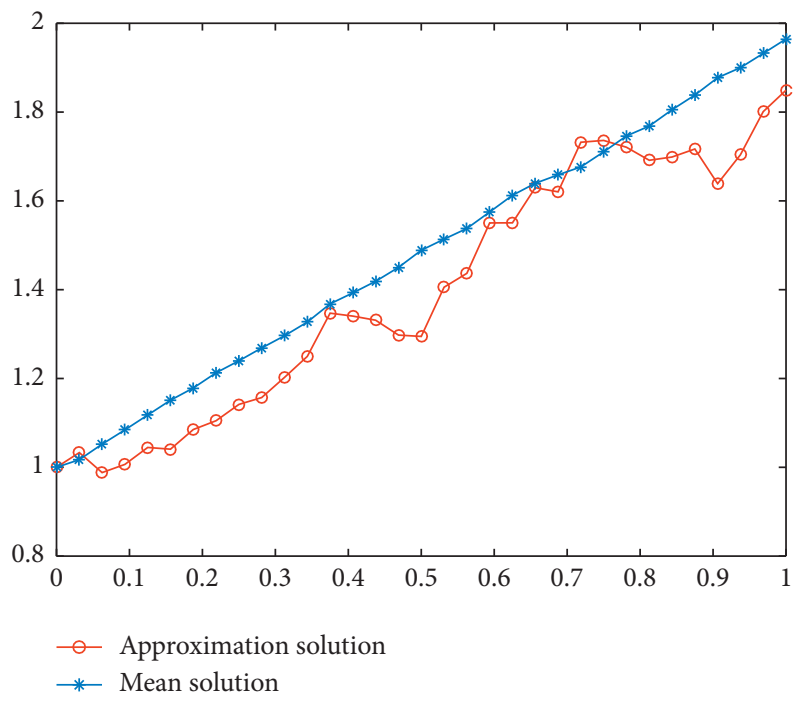

FIGURE 4: $m=2^{5}$, the approximate solution and mean solution for Example 2 . 
(ii)

$$
\begin{aligned}
& \mathbb{E}\left[\min _{c_{1}, \ldots, c_{m}}\left\|X_{m}(t)-f(t)-\int_{0}^{t} \widetilde{S}(u, t) X_{m}(u) \mathrm{d} u-\sum_{k=1}^{q} \int_{0}^{t} \widehat{S}_{k}(u, t) X_{m}(u) \mathrm{d} B_{k}(u)\right\|_{L^{2}}^{2}\right] \\
& \leq \mathbb{E}\left[\left\|\widehat{X}_{m}(t)-f(t)-\int_{0}^{t} \widetilde{S}(u, t) \widehat{X}_{m}(u) \mathrm{d} u-\sum_{k=1}^{q} \int_{0}^{t} \widehat{S}(u, t) \widehat{X}_{m}(u) \mathrm{d} B_{k}(u)\right\|_{L^{2}}^{2}\right] \\
& \leq T^{2}\left\|\widehat{X}_{m}(t)-f(t)-\int_{0}^{t} \widetilde{S}(u, t) \widehat{X}_{m}(u) \mathrm{d} u-\sum_{k=1}^{q} \int_{0}^{t} \widehat{S}(u, t) \widehat{X}_{m}(u) \mathrm{d} B_{k}(u)\right\|_{\infty, E}^{2} \\
& =T^{2}\left\|\widehat{X}_{m}(t)-X(t)+\int_{0}^{t} \widetilde{S}(u, t)\left(X(u)-\widehat{X}_{m}(u)\right) \mathrm{d} u+\sum_{k=1}^{q} \int_{0}^{t} \widehat{S}(u, t)\left(X(u)-\widehat{X}_{m}(u)\right) \mathrm{d} B_{k}(u)\right\|_{\infty, E}^{2} \\
& \leq 3 T^{2}\left(\left\|\widehat{X}_{m}(t)-X(t)\right\|_{\infty, E}^{2}+\left\|\int_{0}^{t} \widetilde{S}(u, t)\left(X(u)-\widehat{X}_{m}(u)\right) \mathrm{d} u\right\|_{\infty, E}^{2}+\left\|\sum_{k=1}^{q} \int_{0}^{t} \widehat{S}(u, t)\left(X(u)-\widehat{X}_{m}(u)\right) \mathrm{d} B_{k}(u)\right\|_{\infty, E}^{2}\right) \\
& \leq 3 T^{2}\left\|\widehat{X}_{m}(t)-X(t)\right\|_{\infty, E}^{2}+3 T^{2} M^{2}\left\|X(t)-\widehat{X}_{m}(t)\right\|_{\infty, E}^{2} \\
& +3 T^{2}\left\|\sum_{k=1}^{q} \int_{0}^{t} \widehat{S}(u, t)\left(X(u)-\widehat{X}_{m}(u)\right) \mathrm{d} B_{k}(u)\right\|_{\infty, E}^{2} \cdot
\end{aligned}
$$

According to isometry property and Doob's inequality,

$$
\begin{aligned}
& \left\|\sum_{k=1}^{q} \int_{0}^{t} \widehat{S}(u, t)\left(X-\widehat{X}_{m}\right)(u) \mathrm{d} B_{k}(u)\right\|_{\infty, E}^{2} \\
& =\mathbb{E}\left[\sup _{0 \leq \tau \leq t}\left|\sum_{k=1}^{q} \int_{0}^{\tau} \widehat{S}(u, t)\left(X(u)-\widehat{X}_{m}(u)\right) \mathrm{d} B_{k}(u)\right|^{2}\right] \\
& \leq 4 \mathbb{E}\left[\left|\sum_{k=1}^{q} \int_{0}^{t} \widehat{S}(u, t)\left(X(u)-\widehat{X}_{m}(u)\right) \mathrm{d} B_{k}(u)\right|^{2}\right] \\
& \leq 4 \mathbb{E} q\left[\sum_{k=1}^{q}\left|\int_{0}^{t} \widehat{S}(u, t)\left(X(u)-\widehat{X}_{m}(u)\right) \mathrm{d} B_{k}(u)\right|^{2}\right] \\
& =4 \mathbb{E} q^{2}\left[\int_{0}^{t}|\widehat{S}(u, t)|^{2}\left|X(u)-\widehat{X}_{m}(u)\right|^{2} \mathrm{~d} u\right] \\
& \leq 4 T M^{2} q^{2}\left\|X(t)-\widehat{X}_{m}(t)\right\|_{\infty, E}^{2} .
\end{aligned}
$$

By (41)-(43), we get

$$
\begin{aligned}
& \mathbb{E}\left[\min _{c_{1}, \ldots, c_{m}}\left\|X_{m}(t)-f(t)-\int_{0}^{t} \widetilde{S}(u, t) X_{m}(u) \mathrm{d} u-\sum_{k=1}^{q} \int_{0}^{t} \widehat{S}_{k}(u, t) X_{m}(u) \mathrm{d} B_{k}(u)\right\|_{L^{2}}^{2}\right] \\
& \leq\left(3 T^{2}+3 T^{4} M^{2}+12 T^{3} M^{2} q^{2}\right)\left\|\widehat{X}_{m}(t)-X(t)\right\|_{\infty, E}^{2} \\
& \leq\left(3 T^{2}+3 T^{4} M^{2}+12 T^{3} M^{2} q^{2}\right) \omega(X, h) \longrightarrow 0,
\end{aligned}
$$


and the proof of the theorem is completed.

\section{Numerical Examples}

The following two examples show the availability and accuracy of the method mentioned in Section 4. The algorithms were implemented by MATLAB 2016a.

Example 1. The linear SIVIE is studied as follows:

$$
\begin{aligned}
X(t)= & X_{0}+\int_{0}^{t} r X(u) \mathrm{d} u \\
& +\sum_{k=1}^{q} \int_{0}^{t} a_{k} X(u) \mathrm{d} B_{k}(u), \quad u, t \in[0,1),
\end{aligned}
$$

where $X(t)=X_{0} e^{\left(r-(1 / 2) \sum_{k=1}^{q} a_{k}^{2}\right) t+\sum_{k=1}^{q} a_{k} B_{k}(t)}, 0 \leq t<1$, and $B_{k}(u)$ are $q$-dimensional Brownian motions. When $X_{0}=1 / 200, r=1 / 20, a_{1}=1 / 50, a_{2}=2 / 50, a_{3}=4 / 50, \quad$ and $a_{4}=9 / 50$, the numerical results are shown in Tables 1 and 2 . The trajectories are shown in Figures 1 and 2, where $n=100$ is the number of iterations.

We can see from Figures 1 and 2 and Tables 1 and 2 that the approximate solution and the exact solution are very close. This method is more accurate than the method in [30], and this method is feasible and effective for solving the $q$ dimensional SIVIEs.

Example 2. The linear SIVIE is studied as follows:

$$
\begin{aligned}
X(t)= & X_{0}+\int_{0}^{t} e^{-(t-u)} X(u) \mathrm{d} u \\
& +\sum_{k=1}^{q} \int_{0}^{t} a_{k} e^{-(t-u)} X(u) \mathrm{d} B_{k}(u), \quad u, t \in[0,1),
\end{aligned}
$$

where $B_{k}(u)$ are q-dimensional Brownian motions. When $X_{0}=1, a_{1}=1 / 50, a_{2}=2 / 50, a_{3}=4 / 50$, and $a_{4}=9 / 50$, the trajectories are shown in Figures 3 and 4.

\section{Conclusion}

In this paper, a numerical method based on the least squares method and BPFs is proposed to solve multidimensional linear SIVIEs different from that of Maleknejad et al., who only used BPFs to solve equations [30]. The least squares method is a mathematical optimization technique that seeks the best function match of data by minimizing errors and quickly obtaining unknown coefficients. Combining it with BPFs can make the error of the numerical solution of multidimensional linear SIVIEs smaller. In Section 6, the numerical simulations are carried out and compared with the literature of [30]; this paper concludes that this method is better than applying only the block pulse functions directly.

\section{Data Availability}

The data used to support the findings of this study are included within the article.

\section{Conflicts of Interest}

The authors declare that they have no conflicts of interest.

\section{Acknowledgments}

This paper was supported by the NSF Grant 2016CFB526 of Hubei Province.

\section{References}

[1] X. Wen and J. Huang, "A numerical method for linear stochastic ito-volterra integral equation driven by fractional brownian motion," in Proceedings of the 2019 IEEE International Conference on Artificial Intelligence and Computer Applications (ICAICA), pp. 121-125, Dalian, China, March 2019.

[2] M. Namik Oğuztöreli, “Time-lag control systems," Jornal Brasileiro de Nefrologia, vol. 32, 1966.

[3] J. J. Levin and J. A. Nohel, "On a system of integrodifferential equations occurring in reactor dynamics. II," Archive for Rational Mechanics and Analysis, vol. 11, no. 1, pp. 210-243, 1962.

[4] M. Khodabin, K. Maleknejad, M. Rostami, and M. Nouri, "Interpolation solution in generalized stochastic exponential population growth model," Applied Mathematical Modelling, vol. 36, no. 3, pp. 1023-1033, 2012.

[5] J. Zhang, Y. Li, and J. Xie, "Numerical simulation of fractional control system using Chebyshev polynomials," Mathematical Problems in Engineering, vol. 2018, pp. 1-5, 2018.

[6] A. Boggess and F. J. Narcowich, A First Course in Wavelets with Fourier Analysis, Publishing House of Electronics Industry, Beijing, China, 2002.

[7] D. S. Mohamed and R. A. Taher, "Comparison of Chebyshev and Legendre polynomials methods for solving two dimensional Volterra-fredholm integral equations," Journal of the Egyptian Mathematical Society, vol. 25, no. 3, pp. 302-307, 2017.

[8] X. Y. Sang, G. Jiang, J. H. Wu, and Y. Y. Lu, "Numerical solution of nonlinear stochastic Itô-Volterra integral equations by block pulse functions," Mathematica Applicata, vol. 32, no. 4, pp. 935-946, 2019, in Chinese.

[9] Z. Nikooeinejad and M. Heydari, "Nash equilibrium approximation of some class of stochastic differential games: a combined Chebyshev spectral collocation method with policy iteration," Journal of Computational and Applied Mathematics, vol. 362, 2019.

[10] R. Ezzati and S. Najafalizadeh, "Application of Chebyshev polynomials for solving nonlinear Volterra-Fredholm integral equations system and convergence analysis," Indian Journal of Science and Technology, vol. 5, no. 2, pp. 2060-2064, 2012.

[11] K. Maleknejad, B. Basirat, and E. Hashemizadeh, "Hybrid Legendre polynomials and Block-Pulse functions approach for nonlinear Volterra-Fredholm integro-differential equations," Computers \& Mathematics with Applications, vol. 61, no. 9, pp. 2821-2828, 2011.

[12] K. Maleknejad, M. Shahrezaee, and H. Khatami, "Numerical solution of integral equations system of the second kind by Block-Pulse functions," Applied Mathematics and Computation, vol. 166, no. 1, pp. 15-24, 2005.

[13] K. Maleknejad, M. Khodabin, and M. Rostami, "Numerical solution of stochastic Volterra integral equations by a stochastic operational matrix based on block pulse functions," 
Mathematical and Computer Modelling, vol. 55, no. 3-4, pp. 791-800, 2012.

[14] M. Asgari, E. Hashmemizadeh, M. Khodabin et al., "Numerical solution of nonlinear stochastic integral equation by stochastic operational matrix based on Bernstein polynomials," Bulletin mathématique de la Société des Sciences Mathématiques de Roumanie, vol. 57, no. 1, pp. 3-12, 2014.

[15] J. H. Wu, G. Jiang, and X. Y. Sang, "Numerical solution of nonlinear stochastic Itô-Volterra integral equations based on Haar wavelets," Advances in Difference Equations, vol. 503, pp. 1-12, 2019.

[16] F. Mirzaee, S. Alipour, and N. Samadyar, "Numerical solution based on hybrid of block-pulse and parabolic functions for solving a system of nonlinear stochastic Itô-Volterra integral equations of fractional order," Journal of Computational and Applied Mathematics, vol. 349, pp. 157-171, 2019.

[17] P. E. Kloeden and E. Platen, Numerical Solution of Stochastic Differential Equations, Springer-Verlag, Berlin, Germany, 1999.

[18] Z. Tang, E. Tohidi, and F. He, "Generalized mapped nodal Laguerre spectral collocation method for Volterra delay integro-differential equations with noncompact kernels," Computational and Applied Mathematics, vol. 39, no. 4, pp. 1-22, 2020.

[19] F. Mirzaee, N. Samadyar, and S. F. Hoseini, "Euler polynomial solutions of nonlinear stochastic Itô-Volterra integral equations," Journal of Computational and Applied Mathematics, vol. 330, no. 330, pp. 574-585, 2018.

[20] F. Mirzaee and S. Alipour, "Cubic B-spline approximation for linear stochastic integro-differential equation of fractional order," Journal of Computational and Applied Mathematics, vol. 366, Article ID 112440, 2019.

[21] W. F. Blyth, R. L. May, and P. Widyaningsih, "Volterra integral equations solved in Fredholm form using Walsh functions," ANZIAM Journal, vol. 45, pp. 269-282, 2003.

[22] X. Dai and A. Xiao, "Levy-driven stochastic Volterra integral equations with doubly singular kernels: existence, uniqueness, and a fast EM method," Advances in Computational Mathematics, vol. 46, no. 2, pp. 1-23, 2020.

[23] X. Dai, W. Bu, and A. Xiao, "Well-posedness and EM approximations for non-Lipschitz stochastic fractional integrodifferential equations," Journal of Computational and Applied Mathematics, vol. 356, pp. 377-390, 2019.

[24] S. Alipour and F. Mirzaee, "An iterative algorithm for solving two dimensional nonlinear stochastic integral equations: a combined successive approximations method with bilinear spline interpolation," Applied Mathematics and Computation, vol. 371, 2020.

[25] M. Saffarzadeh, G. B. Loghmani, and M. Heydari, "An iterative technique for the numerical solution of nonlinear stochastic Itô -Volterra integral equations," Journal of Computational and Applied Mathematics, vol. 333, pp. 74-86, 2018.

[26] M. Saffarzadeh, M. Heydari, and G. Barid Loghmani, "Convergence analysis of an iterative algorithm to solve system of nonlinear stochastic Itô-Volterra integral equations," Mathematical Methods in the Applied Sciences, vol. 43, no. 8, pp. 5212-5233, 2020.

[27] M. Saffarzadeh, M. Heydari, and G. B. Loghmani, "Convergence analysis of an iterative numerical algorithm for solving nonlinear stochastic Itô-Volterra integral equations with m-dimensional Brownian motion," Applied Numerical Mathematics, vol. 146, pp. 182-198, 2019.
[28] G. Jiang, X. Sang, J. Wu, and B. Li, "Numerical solution of two-dimensional nonlinear stochastic itô-volterra integral equations by applying block pulse functions," Advances in Pure Mathematics, vol. 09, no. 02, pp. 53-66, 2019.

[29] M. Ahmadinia, H. AfshariA, and M. Heydari, "Numerical solution of Itô-Volterra integral equation by least squares method," Numerical Algorithms, vol. 84, no. 3, 2020.

[30] K. Maleknejad, M. Khodabin, and M. Rostami, "A numerical method for solving m-dimensional stochastic Itô-Volterra integral equations by stochastic operational matrix," Computers \& Mathematics with Applications, vol. 63, pp. 133-143, 2012.

[31] Z. H. Jiang and W. Schaufelberger, Block Pulse Functions and Their Applications in Control Systems, Springer, Berlin, Germany, 1992.

[32] T. J. Rivlin, An Introduction to the Approximation of Functions, Dover Publications, New York, NY, USA, 1981. 